\title{
Total Anomalous Systemic Venous Drainage to the Left Atrium: A Rare Case of Left Atrial Hysomerism without Intra-Cardiac Defect
}

Del Gaizo F*, Caiainiello G, Palladino MT, Santoro G and Russo MG

Department of Pediatric Cardiology and of Pediatric Cardiac Surgery, AORN dei Colli Monaldi Hospital, Naples, Italy

*Corresponding author: Del Gaizo F, Department of Pediatric Cardiology and of Pediatric Cardiac Surgery, AORN dei Colli Monaldi Hospital, Naples, Italy, Tel: 0393382565187; E-mail: fofi571@hotmail.it

Rec date: Nov 27, 2014; Acc date: Jan 22, 2015; Pub date: Jan 24, 2015

Copyright: ( 2015 Gaizo FD, et al. This is an open-access article distributed under the terms of the Creative Commons Attribution License, which permits unrestricted use, distribution, and reproduction in any medium, provided the original author and source are credited.

Keywords: Coronary sinus; Vena cava superior; Echocardiography

\section{Introduction}

Total anomalous systemic venous return is a very rare malformation, where the vena cava inferior (IVC), the vena cava superior (SVC), and coronary sinus drain into left atrium. In this case, we report an anomaly characterized by a left atrial isomerism with anomalous drainage of IVC, SVC, SSVC, and coronary sinus into left atrium in a small female-baby.

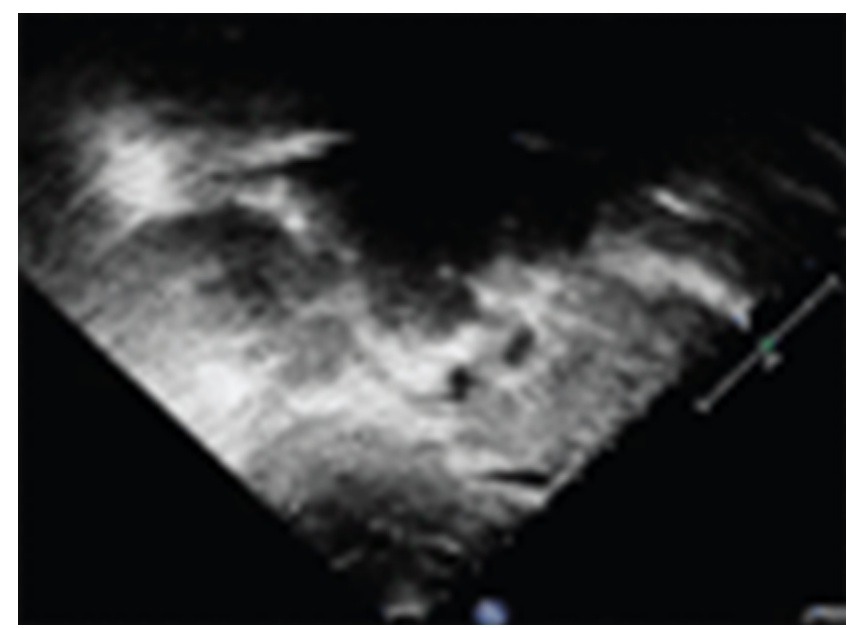

Figure 1: Two-dimensional (2D) echocardiography with substernal coronal view showing the relationship of the aorta and inferior vena cava (IVC) suggestive of left isomerism

\section{Case Report}

We reported the history of S. She received fetal diagnosis of "total anomalous systemic venous connection TASVC and persistent left superior vena cava draining to left atrium"

\section{Personal history}

She was born at $38^{\circ} \mathrm{w}$, weight $\mathrm{kg} 2.860$, she was cianotyc at birth (oxymetry sat. about 70\%). At 3 days of life she was submitted to an external echocardiography (Figure 1 ) and a cardiac catheterization, at our Paediatric Cardiology, which showed situs visceral solitus and left anatomical conformation of both atria and confirmed TASVC (Figures 2-4).

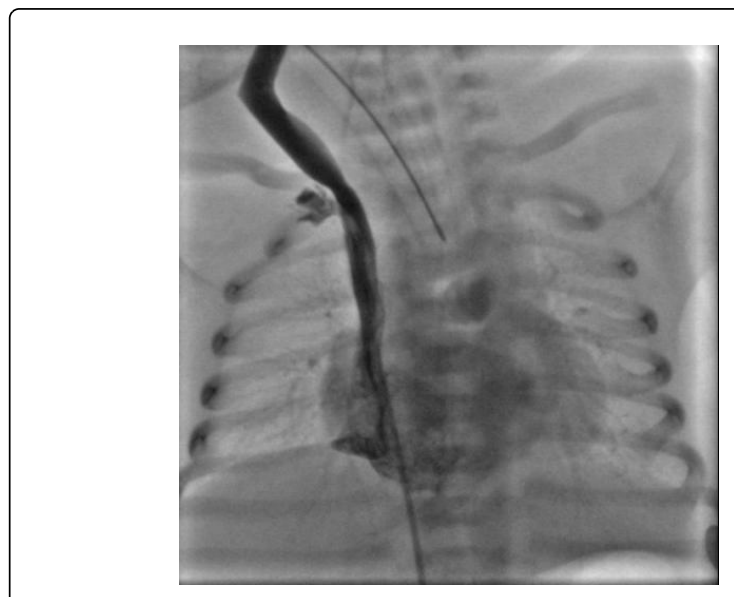

Figure 2: Conformation of RVCS

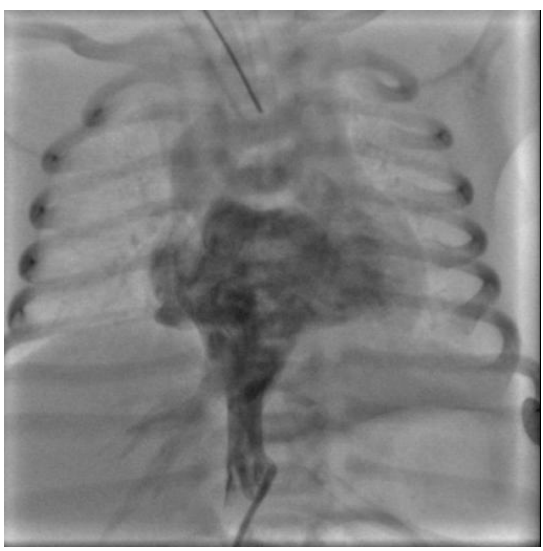

Figure 3: Confirmation of VCI

\section{Surgery intervention}

At 4 days of life we performed a surgical intervention with atrial septostomy a and outpatient clinical observations report perioral cyanosis, oxymetry sat was $80 \%$ and adequate atrial blood mixing at echocardiography. During months we observed the patient with no clinic variations.

When she was 10 months old, she was exposed to a surgical atrial septal reconstruction with bovine pericardial patch draining the vena 
Citation: Del Gaizo F, Caiainiello G, Palladino MT, Santoro G, Russo MG (2015) Total Anomalous Systemic Venous Drainage to the Left Atrium: A Rare Case of Left Atrial Hysomerism without Intra-Cardiac Defect. J Vasc Med Surg 3: 172. doi:10.4172/2329-6925.1000172

Page 2 of 2

cava to the right side, she received a hypothermic CEC and a Custodiol Cardioplegia (Figure 5 and 6).

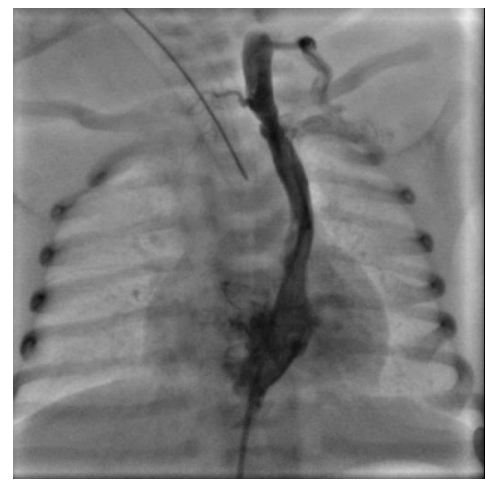

Figure 4: Confirmation of SSVC

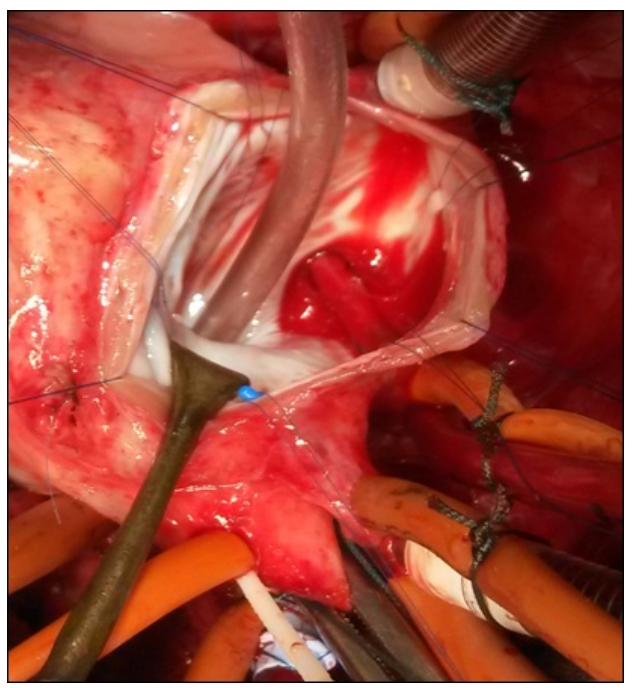

Figure 5: Surgical view of IVC, SVC, SSVC

\section{Follow up}

During the Postoperative she was subjected to echocardiographic controls and electrocardiograms. Echocardiography controls showed a good surgical outcome and small septal defect with L-R shunt, the electrocardiograms observed a postoperative complication whit junctional rhythm, confirmed by 24-hour Holter. After surgical intervention she took therapy with Cardioaspirin for 6 months.

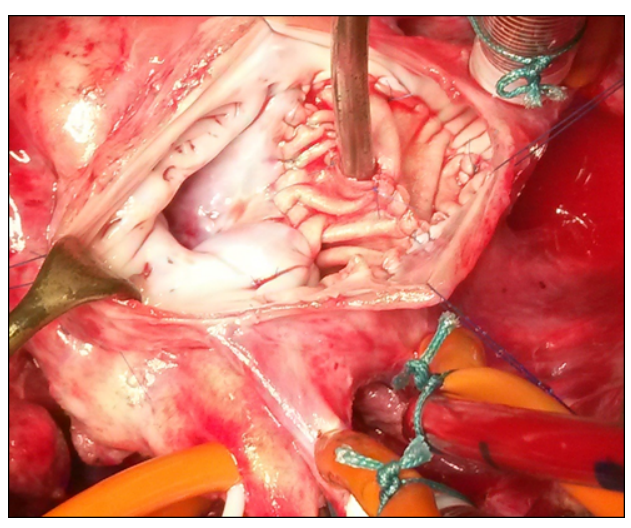

Figure 6: Patch draining the vena

\section{Conclusion}

S. is now a 15 months old little girl in good clinical conditions (oxymetry sat. 85\%), echocardiography controls confirmed a good surgical outcome and there were no further arrhythmias evaluated with Holter. 